\title{
Stratégies de lecture exogénétiques : approches de l'intertextualité invisible
}

Dirk Van Hulle

\section{OpenEdition}

1 Journals

Édition électronique

URL : https://journals.openedition.org/genesis/5602

DOI : 10.4000/genesis.5602

ISSN : 2268-1590

Éditeur :

Presses universitaires de Paris Sorbonne (PUPS), Société internationale de génétique artistique littéraire et scientifique (SIGALES)

Édition imprimée

Date de publication : 15 décembre 2020

Pagination : 29-44

ISBN : 979-10-231-0704-3

ISSN : 1167-5101

\section{Référence électronique}

Dirk Van Hulle, «Stratégies de lecture exogénétiques : approches de l'intertextualité invisible », Genesis [En ligne], 51 | 2020, mis en ligne le 20 décembre 2021, consulté le 13 mars 2023. URL : http:// journals.openedition.org/genesis/5602 ; DOI : https://doi.org/10.4000/genesis.5602 


\section{Stratégies de lecture exogénétiques : approches de l'intertextualité invisible}

Dirk Van Hulle

$\mathrm{D}$ epuis que le terme «intertextualité » a été inventé par Julia Kristeva dans les années 1960, divers chercheurs lui ont donné de nombreuses significations différentes, à tel point que Kristeva a elle-même pris ses distances avec cette notion moins d'une décennie après son introduction 1 . S'appuyant sur la notion de «dialogue» de Mikhaïl Bakhtine, Kristeva soutient que «tout texte se construit comme mosaïque de citations, tout texte est absorption et transformation d'un autre texte ${ }^{2} »$. De même, Roland Barthes déclarait que chaque texte appartient à l'intertextuel ${ }^{3}$. Selon Kristeva, «l'intertextualité» désignait la manière dont les textes (littéraires ou non) émergent d'un ordre sémiotique particulier, ce qui implique des présuppositions sémiotiques complexes ${ }^{4}$, et Jonathan Culler affirmait que l'intertextualité est «less a name for a work's relation to particular prior texts than a designation of its participation in the discursive space of a culture ${ }^{5}$ ». Dans les années 1980 et 1990, des théoriciens comme Gérard Genette et Michael Riffaterre ont recalibré la notion d'«intertextualité». Genette la concevait comme une relation de «co-présence» dans une

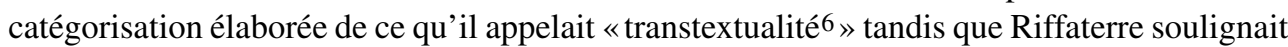
que l'intertextualité était plutôt une question de lecture que d'écriture ${ }^{7}$. Plus récemment, Daniel Ferrer a proposé une approche centrée sur «le fonctionnement intertextuel» plutôt que sur l'intertexte lui-même 8 .

Dans le contexte de ce débat théorique, le présent article tente d'évaluer les tensions et les correspondances entre exogenèse et intertextualité, en abordant notamment le phénomène selon lequel un intertexte agit sur la genèse d'un texte, mais reste invisible dans sa version publiée - ce qu'on pourrait appeler l'intertextualité invisible. J'examine dans quelle mesure l'exogenèse diffère de la «critique des sources» et propose quelques stratégies de lecture exogénétiques pour traiter de l'intertextualité invisible.

1. Julia Kristeva, La Révolution du langage poétique, Paris, Seuil, 1974, p. 59-60.

2. Julia Kristeva, «Le mot, le dialogue et le roman», Sèméiotikè. Recherche pour une sémanalyse, Paris, Seuil, 1969, p. 85 .

3. Roland Barthes, «From Work to Text», dans J.V. Harari (éd.), Textual Strategies, Ithaque, Cornell UP, 1979 , p. $73-81 ;$ p. 77

4. Julia Kristeva, «The Bounded Text», dans L.S. Roudiez (éd.), Desire in Language, New York, Columbia UP, 1980 , p. $37-38$

5. Jonathan Culler, «Presupposition and Intertextuality», MLN, vol. XCI, no 6, 1976, p. 1380-1396; p. 1382. Ma traduction : «plutôt une désignation de la participation d'une œuvre à l'espace discursif de la culture qu'un nom pour sa relation avec des textes antérieurs particuliers ».

6. Gérard Genette, Palimpsestes. La Littérature au second degré, Paris, Seuil, 1982, p. 8.

7. Michael Riffaterre, «La Trace de l'intertexte», Pensée, no 215, 1980, p. 4-18.

8. Daniel Ferrer, «Quelques remarques sur le couple intertextualité-genèse», dans P. Gifford et M. Schmid (éd.),

La Création en acte. Devenir de la critique génétique, Amsterdam, Rodopi, 2007, p. 205-216; p. 206. 
Critique de la critique des sources : influence versus intertextualité
$\mathrm{Si}$, du point de vue de Kristeva, le terme «intertextualité» a été détourné et «souvent entendu dans le sens banal de "critique des sources" d'un texte ${ }^{9}$ », il y a bien sûr des raisons à ce phénomène de détournement dans la critique littéraire. L'une d'entre elles est que bon nombre des termes traditionnels utilisés pour désigner les interrelations textuelles (comme «source», «influence» ou «emprunt») impliquent une hiérarchie, ce qui suggère que le texte postérieur est dérivé et non original, tandis que «l'intertextualité» a l'avantage de ne pas suggérer cette inégalité 10 .

Il ne s'agit d'ailleurs pas d'une question exclusivement littéraire. La critique de Michael Baxandall à l'égard du terme «influence » (et implicitement du terme «source») est toujours pertinente, tant en histoire de l'art qu'en études littéraires. L' «influence, écrit-il, est une malédiction de la critique d'art notamment à cause de ses préjugés grammaticaux erronés sur qui est l'agent et qui est le patient ${ }^{11}{ }$. Au début du Xxi ${ }^{\mathrm{e}}$ siècle, Scarlett Baron résume brièvement la situation comme «un choix entre deux paradigmes contradictoires : l'influence d'une part, l'intertextualité d'autre part 12 ».

Une exposition des appropriations «intertextuelles» de Rubens (au Musée Städel à Francfort ${ }^{13}$ ) a montré comment l'artiste avait progressivement traité sa propre interprétation et esquisse d'une sculpture d'un centaure pour ensuite l'utiliser afin de donner forme à une peinture sur un thème religieux, Pilate présentant le Christ à la foule («Ecce Homo»). Le tableau gagne en complexité à travers cette perspective «intertextuelle». Il montre non seulement un Christ au torse de centaure, mais l'ensemble de la transformation d'un thème mythologique grec en un thème chrétien, ce qui s'avère un processus complexe, dans lequel les brouillons et esquisses de l'artiste jouent un rôle intermédiaire crucial. D'une façon similaire, dans les études littéraires, les cahiers et les brouillons d'un écrivain jouent un rôle central dans l'analyse des relations intertextuelles. Il est révélateur que le titre de l'exposition «Le pouvoir de la transformation » comporte les mêmes accents sur l'opération, le fonctionnement et le traitement des intertextes que ceux préconisés par Ferrer ${ }^{14}$.

Au cours des dernières années, les nouveaux développements dans les humanités numériques, notamment dans le domaine des logiciels de «réutilisation de texte», ont rendu le débat sur l'influence/intertextualité plus pertinent que jamais. Récemment, Dennis McCarthy et June Schlueter ont présenté les résultats de leurs recherches en utilisant un logiciel de plagiat sur les œuvres de Shakespeare, relevant plusieurs correspondances entre un manuscrit de 1576 de George North et plus de vingt passages de Shakespeare 15. C'est précisément dans le contexte de ces développements numériques qu'il est important de réévaluer la notion

9. Julia Kristeva, La Révolution du langage poétique, op. cit., p. 59-60.

10. Gregory Machacek, «Allusion», PMLA, vol. CXXII, no 2, 2007, p. 522-536; p. 525.

11. Michael Baxandall, Patterns of Intention. On the Historical Explanation of Pictures, New Haven, Yale UP, 1985, p. $58-59$

12. Scarlett Baron, «Strandentwining Cables». Joyce, Flaubert, and Intertextuality, Oxford, OUP, 2012 , p. 8.

13. «Rubens, le pouvoir de la transformation», Musée Städel à Francfort, de janvier à mai 2018.

14. Voir note 8 .

15. Alison Flood, «Plagiarism software pins down new source for Shakespeare's plays », The Guardian, 9 février 2018, en ligne sur theguardian.com. 
d'intertextualité, car les nuances du débat influence/intertextualité ne sont pas toujours prises en compte dans les humanités numériques ${ }^{16}$.

Scarlett Baron remarque à juste titre qu' «il est pratiquement impossible de tracer une ligne de démarcation nette et durable entre les concepts d'influence et d'intertextualité17 ». Lorsque Julia Kristeva a renoncé à sa propre terminologie et a préféré travailler avec la notion de «transposition», elle a clairement évité le terme «texte» et s'est distanciée de la «critique des sources». Le terme allemand Quellenforschung (recherche de sources) - qui est toujours d'usage - implique la même métaphore de «sources », qui suppose une origine, une influence qui s'étend ou agit sur un autre texte, rappelant l'action et la causalité qui définissent la notion d'influence selon Susan Stanford Friedman ${ }^{18}$. Ce réflexe philologique ad fontes implique une approche «en amont» de la production littéraire, qui correspond, du moins à première vue, à la «philologie radicale» proposée par Geert Lernout :

Une philologie radicale limite la recherche au «désir-de-dire» originel de toute forme d'écriture et à sa participation à un contexte saturable et restrictif. Si elle ne le faisait pas, elle perdrait toute pertinence. Retirez l'intention et le contexte, et la seule chose qu'il reste à dire à propos d'un texte est qu'il peut signifier tout et son contraire ${ }^{19}$.

On peut reconnaître ce «contexte restrictif» dans d'autres théories de l'intertextualité. Alors que Kristeva évitait la «critique des sources», d'autres ont souligné que sa notion d'intertextualité avait aussi des inconvénients, notamment son applicabilité limitée dans la pratique réelle de la recherche. Lorsque Gérard Genette a introduit sa version de l'intertextualité dans Palimpsestes (1982), il la présentait comme l'un des cinq types de «transtextualité », qui mettent chacun un texte «en relation, manifeste ou secrète, avec d'autres textes $20 »$. C'est précisément parce qu'il était conscient de l'interprétation large et donc vague de l'intertextualité de Kristeva que Genette a présenté sa version comme plus «restrictive $21 »$.

Le concept d'intertextualité de Michael Riffaterre, tel que défini dans «La trace de l'intertexte» (1980), mettait en avant le rôle du lecteur. L'intertextualité, selon Riffaterre, est «la perception, par le lecteur, de rapports entre une œuvre et d'autres, qui l'ont précédée ou suivie 22 ». La «trace» du titre de son essai ne fait pas référence aux traces de lecture d'un

16. Voir par exemple M. Büchler, P. R. Burns, M. Müller, E. Franzini, G. Franzini, «Measuring the Influence of a Work by Text Re-Use», dans C. Biemann et A. Mehler (éd.), Text Mining, Theory and Applications of Natural Language Processing, Springer International Publishing Switzerland, 2014.

17. Scarlett Baron, op. cit., p. 9.

18. Susan Stanford Friedman, «Weavings : Intertextuality and the (Re) Birth of the Author», dans J. Clayton et E. Rothstein (éd.), Influence and Intertextuality in Literary History, Madison, The University of Wisconsin Press, 1991, p. 152 : «It presumes a source, an origin, an agency that flows into or acts upon another.»

19. Geert Lernout, «The Finnegans Wake Notebooks and Radical Philology », D. Hayman et S. Slote (éd.), Probes : Genetic Studies in Joyce, Amsterdam, Rodopi, 1995, p. 19-48; 47 : «A radical philology limits the inquiry to the original desire-to-say of any form of writing and to its participation in a saturable and constraining context. If it did not, it would forfeit all relevance. Take away intention and context, and the only thing left to say about a text is that it can mean anything at all.»

20. Gérard Genette, op. cit., p. 7.

21. Ibid., p. 8.

22. Michael Riffaterre, «La trace de l'intertexte», art. cit., p. 4. 
auteur (comme les notes en marge dans les livres de sa bibliothèque personnelle ou les notes de lecture dans des cahiers), mais aux «structures» et «impératifs textuels». Ces impératifs textuels veillent à ce que les éléments intertextuels soient reconnaissables dans une certaine mesure, ce qui rend ce type d'intertextualité «obligatoire» dans le sens où c'est «une intertextualité que le lecteur ne peut pas ne pas percevoir» en raison d'une certaine incompatibilité avec le contexte ou d'une forme d'agrammaticalité 23 . Selon cette théorie, un «connecteur» sert d'indicateur : «un mot ou un groupe de mots qui est grammatical dans l'intertexte [...] mais $[\ldots]$ agrammatical dans le texte ${ }^{24}$.»

Daniel Ferrer souligne cependant que cette agrammaticalité ne peut guère servir d'outil pour détecter des éléments intertextuels lorsqu'il s'agit d'un texte comme Finnegans Wake de James Joyce. Il montre de manière convaincante comment le «work in progress » de Joyce renverse la théorie de Riffaterre. Les carnets de Finnegans Wake VI.B.42 et VI.B.46 (conservés à l'Université de Buffalo) contiennent des notes de lecture sur Les Aventures de Huckleberry Finn de Mark Twain. Le mot «considerable» a été noté parce qu'il est utilisé comme adverbe - de manière agrammaticale - dans l'intertexte («the nigger was set back considerable»), alors que l'utilisation par Joyce de cette note dans ses brouillons est parfaitement grammaticale (utilisée comme adjectif : «considerable way »)25.

L'édition des carnets de Finnegans Wake à Buffalo montre de nombreux exemples de mots que Joyce s'est appropriés de telle manière qu'il devient parfois impossible - sans recourir aux carnets - de savoir qu'ils proviennent de textes externes. Même si Riffaterre semble être assez sûr qu'un texte garde la mémoire de ses intertextes et que l'érosion intertextuelle est «un faux problème», le texte de Finnegans Wake est tellement saturé de liens intertextuels que ce que Jérôme McGann qualifiait de «condition textuelle26» devient la «condition intertextuelle».

La condition intertextuelle dénote la façon dont un texte montre ou cache ses liens avec d'autres textes. Dès lors, le «code linguistique» et le «code bibliographique», tels que définis par McGann27, ne sont pas les seules caractéristiques d'un texte; il me semble nécessaire d'y ajouter ce que l'on pourrait appeler le «code génétique » du texte. Ce code est particulièrement pertinent dans des cas d'intertextualité invisible. Daniel Ferrer suggère d'élargir les notions d' «intertextualité» et de «lecteur» en incluant des lecteurs généticiens ${ }^{28}$. S'il faut des lecteurs généticiens, ou des lecteurs informés des manuscrits, pour percevoir certains de ces intertextes comme tels, cela nécessite une approche qui consiste en quelques stratégies de lecture exogénétiques, que j'aimerais aborder dans la suite de cet article, en utilisant le cas de l'œuvre de Samuel Beckett.

23. Ibid., p. 4

24. Michael Riffaterre, «Contraintes intertextuelles», É. Le Calvez et M.-C. Canova-Green (éd.), Texte(s) et Intertexte(s), Amsterdam, Rodopi, 1997, p. 38.

25. James Joyce, Finnegans Wake, Londres, Faber and Faber, 1939, p. 317-23; Daniel Ferrer, «Quelques remarques sur le couple intertextualité-genèse», art. cit., p. 209.

26. Jerome J. McGann, The Textual Condition, Princeton, Princeton UP, 1991.

27. Ibid., p. 52.

28. Daniel Ferrer, «Quelques remarques sur le couple intertextualité-genèse», art. cit., p. 213. 
La critique génétique s'est montrée particulièrement utile pour ouvrir la notion d'intertextualité et sensibiliser le lecteur à l'intertextualité des textes d'auteurs modernes. Par exemple, dans le cas de Samuel Beckett, l' «érosion intertextuelle» fait partie intégrante de la poétique de l'ignorance de l'auteur. L'érudition de Beckett était considérable. Jeune homme, il avait reçu une éducation intellectuelle issue de l'esprit encyclopédique des années 1920 et 1930, époque à laquelle Paul Otlet développait son Répertoire Bibliographique Universel, précurseur de Google. Dans cet esprit d'orgueil encyclopédique, Beckett a également acquis des connaissances de toutes sortes, à la fois en tant qu'étudiant et qu'autodidacte. Plus tard, il confia même à Jake Schwartz qu'à un certain moment de sa vie, il avait étudié l'encyclopédie. Mais lorsque Schwartz lui donna la 11e édition de l'Encyclopédie Britannique dans les années 1950, sa poétique avait résolument tourné le dos à l'érudition, comme il le laissa entendre à Jacoba van Velde : «On m'a donné l'édition 1911 de l'Encyclopédie Britannique. 28 volumes. Trop tard 29 .» Écrire en français après la guerre n'était pas seulement une question linguistique; c'était aussi une façon de s'éloigner de la langue et de l'écriture encyclopédique de Joyce. Pourtant, l'érudition de Beckett faisait alors tellement partie intégrante de sa personne que les intertextes n'ont cessé de surgir, même dans ses écrits d'après-guerre. Beckett a donc décidé de déployer activement le phénomène de «l'érosion intertextuelle» comme stratégie d'écriture. Par exemple, dans Oh les beaux jours, Winnie se souvient constamment d'intertextes, mais elle le fait presque systématiquement de manière approximative, comme dans sa déclamation à plusieurs reprises dans la pièce d'une série de vers de poésie, qui contiennent tous le mot «malheur» (woe). D'abord, elle ne se souvient que partiellement d'une réplique de Hamlet 30 :

what are those wonderful lines - (wipes one eye) - woe woe is me - (wipes the other) - to see what I see - (looks for spectacles) - ah yes - (takes up spectacles)

[quels sont ces vers merveilleux? - (elle s'essuie un æill) - malheur à moi - (l'autre æeil) - qui vois ce que je vois - (elle cherche les lunettes $)$ - hé oui - (elle ramasse les lunettes $)^{31}$.

Ensuite, elle utilise le mot bouche-trou «something»/ «ta-la» pour combler ses trous de mémoire d'une phrase du Paradis perdu de John Milton ${ }^{32}$ :

What is that wonderful line? (Lips.) Oh fleeting joys - (lips) - oh something lasting woe

[Quel est ce vers admirable? (Lèvres.) Oh fugitives joies - (lèvres) - oh... ta-la lents malheurs ${ }^{33}$ ].

Et puis elle oublie les mots «moody Madness» dans un vers de Thomas Gray34, utilisant à nouveau «something something» / «ta-la» comme mot bouche-trou :

what is that wonderful line... laughing wild... something something laughing wild amid severe woe. [quel est ce vers merveilleux... ta-la malheur, suffit, tu m'as assez fait rire ${ }^{35}$ ].

29. Samuel Beckett, lettre à Jacoba van Velde, 12 avril 1958; BnF NAF 19794, 53.

30. «Oh, woe is me, / T'have seen what I have seen, see what I see !» (William Shakespeare, Hamlet, III.1, p. 164-165).

31. Samuel Beckett, Oh les beaux jours, Paris, Minuit, 1963, p. 14.

32. «O fleeting joys / Of Paradise, dear bought with lasting woes !» (John Milton, Paradise Lost, Book X, 11. p. 741-742).

33. Samuel Beckett, Oh les beaux jours, op. cit., p. 19.

34. «and moody Madness laughing wild amidst severest woes» (Thomas Gray, «Ode on a Distant Prospect of Eton College »).

35. Samuel Beckett, Oh les beaux jours, op. cit., p. 42.

\section{Érosion intertextuelle et intertextualité invisible dans l'œuvre de Samuel Beckett}


Ici, l'érosion devient partie intégrante du texte et, à l'instar de la condition physique de Winnie (son corps s'enfonçant dans la terre, présentant l'art de vivre plutôt comme l'art de mourir), sa condition intellectuelle et linguistique est une désintégration de la condition intertextuelle de l'individu - intertextuelle au sens large où l'identité de la personne est caractérisée par sa «participation à l'espace discursif de la culture ${ }^{36}$ ». Dans d'autres œuvres de Beckett le jeu de l'érosion intertextuelle est cependant souvent plus subtil que dans Oh les beaux jours et implique parfois une forme d'intertextualité invisible. Pour essayer de lire et interpréter cette intertextualité invisible, il me semble utile de développer un ensemble de stratégies de lecture exogénétiques.

\section{Stratégie 1 : une approche exogénétique directe}

Parfois un intertexte agit sur la genèse mais n'a laissé aucune trace dans le texte publié. Par exemple, dans le manuscrit de Malone meurt, l'histoire de Sapo contient un passage où le maître bat Sapo avec une férule : «Penche-toi, Saposcat, dit le maître. Il n'était pas en colère, il s'acquittait d'une tâche pénible.» Sapo ne bouge pas et dit : «Mon père ne me bat pas. » «C'est à moi qu'il délègue ce pénible office, dit le maître, et avec une vivacité extraordinaire il décerna à Sapo un coup cinglant sur les mollets ${ }^{37}$.» Dans cette scène Beckett cite le vers biblique «Celui qui ménage sa verge hait son fils 38 ». La citation n'a pas été retenue dans la version publiée. Bien que l'omission n'ait peut-être pas de conséquences immédiates, la présence de ce vers biblique dans les manuscrits joue son rôle dans le fonctionnement intertextuel et une lecture exogénétique découvre ainsi une association de la Bible avec une scène de violence, une association qui n'est pas sans importance interprétative. Bien sûr, la question se pose de savoir si une trace exogénétique biffée est une présence ou plutôt une absence. Mais, tout comme les pentimenti dans les arts visuels ${ }^{39}$, ce n'est qu'après avoir découvert la présence d'une connexion intertextuelle qu'on peut commencer à considérer son absence.

\section{Stratégie 2 : une approche endogénétique}

L'endogenèse peut être parfois un moyen de découvrir une dimension intertextuelle. Par exemple, ce passage de Malone meurt : «Après l'échec, la consolation, le repos, je recommençais, à vouloir vivre, faire vivre, être autrui, en moi, en autrui. Que tout ça est faux 40 » est suivi, dans le manuscrit, de cette phrase : «Cependant je n'ai jamais rencontré de frère ${ }^{41}$. » Sans contexte, le mot «frère» peut difficilement être lu comme une allusion intertextuelle. Ce n'est que lorsqu'il est lu conjointement avec la prochaine version - «Je n'ai jamais rencontré de semblable» - que l'allusion devient claire, faisant

36. Jonathan Culler, art. cit., p. 1382.

37. Samuel Beckett, manuscrit de Malone meurt, Beckett Digital Manuscript Project, BDMP5, MS-HRC-SB-7-2, fo $63 \mathrm{r}^{\circ}$.

38. Livre des Proverbes 13:24; Beckett Digital Manuscript Project, BDMP5, MS-HRC-SB-7-2, fo 63 ro.

39. Dirk Van Hulle, «The Pentimenti Principle : The Draft and the Draff in Beckett's Critique of Narrative Reason », Samuel Beckett Today / Aujourd'hui, no 31, 2019, p. 37-52.

40. Samuel Beckett, Malone meurt, Paris, Minuit, 1951, p. 37.

41. Beckett Digital Manuscript Project, BDMP5 MS-HRC-SB-7-2, fo 75 ro 
référence à «Au lecteur» de Baudelaire. Le poème d'ouverture des Fleurs du mal, dont Beckett avait un exemplaire dans sa bibliothèque, se termine par la phrase : «- Hypocrite lecteur, - mon semblable, - mon frère! 42 » Dans ce cas-ci, Beckett joue un remarquable jeu d'intertextualité à travers les versions et c'est grâce à une lecture diachronique et endogénétique que l'intertexte se révèle.

Dès que certains de ces intertextes sont devenus apparents, ils deviennent plus facilement perceptibles dans d'autres œuvres du même auteur. En d'autres termes, l'intertexte se révèle de manière intratextuelle, et pas seulement dans l'endogenèse, mais aussi dans l'épigenèse - la continuation de la genèse après publication ${ }^{43}$. Par exemple, l'ouverture du script de Film de Beckett se lit dans la version originale en anglais de la manière suivante : «All extraneous perception suppressed, animal, human, divine, self-perception maintains in being 44 ». La première tentative de traduction devient : «Perçu de soi subsiste l'être que nul ne perçoit, $n i$ semblable, ni animal, ni Dieu $45^{»}$. Outre l'intéressant renversement de l'ordre (de animal - humain - divin à humain - animal - divin), la traduction de «human» par «semblable» attire l'attention. L'emploi du mot «semblable» pourrait être interprété comme une référence intertextuelle au même poème de Baudelaire, «Au lecteur». Malheureusement, cette interprétation ne peut être qu'éphémère, car la phrase est immédiatement rayée et la version révisée se rapproche beaucoup plus de la version originale en anglais. Ainsi, l'intertexte agit sur la genèse d'un texte, mais reste invisible dans la version publiée, créant ainsi un réseau «souterrain» exogénétique et intratextuel dans la sous-œuvre de l'auteur, reliant les œuvres individuelles de l'auteur sous la surface du texte publié.

\section{Stratégie 3 : une approche intratextuelle}

Parfois, l'intratextualité - c'est-à-dire les liens établis par un lecteur entre diverses œuvres d'un même auteur, selon la définition de Kareen Martel ${ }^{46}$ - peut aider à identifier l'intertextualité. Dans La Dernière Bande, par exemple, Krapp est décrit comme un «wearish old man» («vieil homme avachi»). En soi, c'est une didascalie qui passe inaperçue. Ce qui est intéressant, cependant, c'est que la même expression revient dans les premières œuvres de Beckett. L'adjectif archaïque «wearish» y apparaît plusieurs fois. Dans l'histoire «Echo's Bones» («Les os d'Echo 47 ») et dans le poème «Enueg I», le «vieil homme avachi» est associé à Démocrite ${ }^{48}$. Comme le souligne John Pilling, ce passage s'inspire d'une note du

42. Voir Beckett Digital Library, http://www.beckettarchive.org/library/BAU-FLE-1.html.

43. Dirk Van Hulle, «Modern Manuscripts and Textual Epigenetics: Samuel Beckett's Works between Completion and Incompletion», Modernism/modernity, vol. XVIII, no 4, 2011, p. 801-812.

44. Samuel Beckett, Film, dans Eh Joe and Other Writings, Londres, Faber and Faber, 1967, p. 29-44, p. 31

45. Beckett Digital Manuscript Project, BDMP8, MS-WU-MSS008-2-32-1, fo 01 ro

46. Kareen Martel, «Les notions d'intertextualité et d'intratextualité dans les théories de la réception », Protée, vol. XXXIII, no 1, 2005, p. 93-102.

47. «"Love" said a wearish voice behind him, "turn round my young friend, face this way do, and tell me what you know of that disorder" ", Samuel Beckett, Echo's Bones, M. Nixon (éd.), Londres, Faber and Faber, 2014, p. 15.

48. «I splashed past a little wearish old man, / Democritus, / scuttling along between a crutch and a stick»; Samuel Beckett, «Enueg I», dans S. Lawlor et J. Pilling (éd.), The Collected Poems of Samuel Beckett, Londres, Faber and Faber, 2012, p. 7. 
cahier «Dream» (UoR MS 5000), tiré de L'Anatomie de la mélancolie de Robert Burton49. Jeune écrivain, Beckett a pris environ trois cents notes sur l'œuvre de Burton, dont le discours, selon Angus Gowland, imite à la fois Démocrite et Héraclite, oscillant entre vitupération satirique et lamentation tragique 50 .

Beckett connaissait Démocrite comme le «philosophe du rire » et Héraclite comme «le philosophe des larmes, sombre, obscur» 51 . En essayant de trouver son propre style en tant qu'écrivain, l'artiste, jeune homme, avait non seulement une affinité avec le style de Burton, mais s'identifiait même dans une certaine mesure à cette polarisation entre Démocrite qui rit et Héraclite qui pleure, comme le suggère une lettre à Nuala Costello du 27 février 193452 . Et cette tension est restée une caractéristique de son style, tant dans sa fiction en prose que dans ses œuvres dramatiques, où elle s'exprime dans un penchant pour le tragicomique. (La traduction anglaise par Beckett lui-même de En attendant Godot, par exemple, est explicitement qualifiée de «tragicomédie» dans son sous-titre.) Même vers la fin de sa carrière, lorsque Desmond Egan a demandé à Beckett s'il avait une opinion plus favorable de la tragédie ou de la comédie, Beckett a suggéré que la réponse était une combinaison des deux ${ }^{53}$. Cela met en lumière une note par ailleurs plutôt énigmatique de son carnet de théâtre, où il gardait ses notes pour sa propre mise en scène de sa pièce de théâtre La Dernière Bande : «Hemocritus et Deraclitus, / philosophes muets 54 ». En changeant les initiales des deux philosophes, Beckett suggérait une fois de plus l'interconnectivité de la tragédie et de la comédie dans sa poétique. Ses œuvres sont peut-être sombres, mais il y a souvent une allusion à ce que Gowland appelle la manière de Burton de «parler "en plaisantant" » («speaking "in jest" 55 »). Ainsi, la connexion intratextuelle avec les premières œuvres - par le biais du mot archaïque «avachi »- peut être utile pour récupérer une partie de l'intertextualité invisible dans les œuvres ultérieures.

\section{Stratégie 4 : une approche indirecte via les marginalia}

Si la bibliothèque de l'auteur existe encore, il semble évident à première vue qu'elle peut aider à découvrir des références intertextuelles invisibles, mais ce n'est souvent que de manière très sinueuse ou indirecte que l'intertextualité fait surface. Dans le cas de Beckett, sa bibliothèque a été partiellement conservée et se trouve toujours dans son appartement

49. «little wearish old man (Democritus)»; Samuel Beckett, “Dream” Notebook, J. Pilling (éd.), Reading, University of Reading, 1999, p. 104. Voir «Democritus [...] was a little wearish old man, very melancholy by nature, averse from company in his latter days, and much given to solitariness »; Robert Burton, The Anatomy of Melancholy, F. Dell et P. Jordan-Smith (éd.), New York, Tudor, 1948 [1621], p. 12.

50. Angus Gowland, «The Worlds of Renaissance Melancholy », Robert Burton in Context, Cambridge, Cambridge UP, 2006, 292. Voir aussi Rina Kim, «The Mind-Body Nexus in Beckett's Writing of Love-Melancholy», Samuel Beckett Today / Aujourd'hui, no 29, 2017, p. 37-50.

51. Samuel Beckett, «Philosophy Notes», TCD MS 10967/24 ro.

52. «Well I might do worse than find myself as it were polarised between Democritus and Heraclitus for all eternity, in a place where sighing is out of melancholy and not out of torment »; Samuel Beckett, The Letters of Samuel Beckett, vol. I, M. Dow Fehsenfeld et L. More Overbeck (éd.), Cambridge, Cambridge UP, 2009, p. 185.

53. «Democritus laughed at Heraclitus weeping \& H. wept at D. laughing. Pick yr. fancy ; Beckett, cité dans Laura Salisbury, Samuel Beckett: Laughing Matters, Comic Timing, Édinbourg, Edinburgh University Press, 2012 , p. 230. 54. Samuel Beckett, Theatrical Notebooks 3: Krapp's Last Tape, Londres, Faber and Faber, 1993, p. 247.

55. Angus Gowland, «The Worlds of Renaissance Melancholy», art. cit. 
parisien, comme il l'avait laissée à sa mort en 1989. Notons l'absence de l'œuvre de Voltaire dans cette bibliothèque. Cela ne signifie certainement pas que Beckett n'ait pas lu Voltaire, mais une approche indirecte via les marginalia des livres existants - une sorte de « via negativa»- est nécessaire pour retrouver des traces de lecture. Pour illustrer cette «via negativa», l'exemplaire de Der Pessimismus d'Olga Plümacher dans la bibliothèque peut servir d'exemple 56 . Beckett a écrit quelques petites notes en marge de l'Introduction, dans laquelle - pour expliquer la notion de pessimisme - Plümacher fait référence à la théodicée de Leibniz, qui affirme que le monde réel est le meilleur de tous les mondes possibles ${ }^{57}$. En ce qui concerne ce débat des Lumières, Beckett était décidément du côté de Voltaire, et dans ce livre philosophique sur le pessimisme, le débat thématisé dans Candide, ou l'optimisme semble avoir retenu son attention.

Par exemple, Plümacher note que même les optimistes les plus résolus ne nient pas la réalité de la douleur. C'est pourquoi elle suggère de remplacer «l'optimisme» (superlatif) statique par un «méliorisme» (comparatif) plus dynamique 58 - une philosophie qui affirme qu'en s'immisçant dans les processus naturels, les êtres humains peuvent apporter des progrès et des améliorations à l'état naturel des choses. À côté de ce passage, Beckett a écrit le néologisme «Pejorismus », ce qui serait le contraire, une philosophie qui suggère qu'il n'y a pas de quoi être fier de son humanité, car l'interférence humaine peut provoquer de graves régressions par rapport aux processus naturels. L'hypothèse selon laquelle notre monde serait le pire et non le meilleur de tous les mondes possibles pourrait conduire à un pessimisme statique, mais le «péjorisme» de Beckett est plus dynamique. Il n'implique pas de progrès (progress), ni nécessairement de régression (regress). Beckett préférait le mot «gress », tout court, en raison de son absence de téléologie 59 . Dans un cahier, Beckett a également noté les termes «pejorism / meliorism 60 ». Il ne les a utilisés dans aucune de ses œuvres, mais cela n'implique pas leur insignifiance. Ils sont en réalité cruciaux dans le développement de sa poétique, qui porte clairement une marque voltairienne. Si Voltaire lui-même est étrangement absent, sa présence est néanmoins perceptible, ironiquement, à l'aide de l'une des Bibles de la bibliothèque, notamment la Bible italienne (datant de la période où Beckett a étudié la littérature italienne et française au Trinity College de Dublin) ${ }^{61}$.

Dans cette Bible, il y a quelques passages marqués, où Beckett semble traduire le texte italien en français. Par exemple, dans Ézéchiel 23 («e s’appassionò per quei fornicatori dale membra d'asino, dall'ardor di stalloni ») il écrit dans la marge : «Elle a recherché ceux qui ont le membre, d'un âne, et déchargent comme des chevaux. » Entre parenthèses, il écrit une référence cryptique «(L. d'Am.)» qui devient plus explicite dans d'autres traductions marginales similaires, comme celle du passage d'Ézéchiel 16 («ma tu eri nuda e scoperta ») :

56. Beckett Digital Library, en ligne sur beckettarchive.org.

57. Olga Plümacher, Der Pessimismus in Vergangenheit und Gegenwart : Geschichtliches und Kritisches, Heidelberg, Georg Weiss Verlag, 1888, p. 2.

58. Ibid.

59. Samuel Beckett, The Letters of Samuel Beckett, vol. I, M. Dow Fehsenfeld et L. More Overbeck (éd.), Cambridge, Cambridge UP, 2009, p. 186.

60. "Whoroscope" Notebook, UoR MS 3000, 45 vo.

61. La Sacra Bibbia, Roma, Società Biblica Britannica e Forestiera, 1924. Beckett Digital Library, en ligne sur beckettarchive.org. 
«Tu as ouvert tes cuisses à tous les passants », suivie de «(Lettres d'Amabed)» entre parenthèses. Beckett a donc apparemment lu cet ouvrage de Voltaire, notamment les notes de bas de page de la «Troisième lettre du journal d'Amabed», qui offre la clé pour reconstruire le processus de lecture de Beckett. Dans le texte de Voltaire, le narrateur non chrétien exprime son étonnement devant le contenu de l'Ancien Testament :

Ce qui me fait le plus de peine, c'est que le dieu de ces gens-là ordonne à un de ses prédicateursa de manger de la matière louable sur son pain; et à un autre, de coucher pour de l'argent avec des filles de joieb, et d'en avoir des enfants ${ }^{62}$.

Dans les notes de bas de page, Voltaire note soigneusement les références aux nombreux passages obscènes de la Bible, puis continue :

Il y a bien pis. Ce savant homme nous a fait remarquer deux sœurs, Oolla et Oolibac. Tu les connais bien, puisque tu as tout lu. Cet article a fort scandalisé ma femme : le blanc de ses yeux en a rougi. J'ai remarqué que la bonne Déra était tout en feu à ce paragraphe63.

Dans la note de bas de page liée à «Oolla et Ooliba», Voltaire cite exactement les passages d'Ézéchiel que Beckett a recopiés en marge de sa Bible. Sa lecture très spécifique de Voltaire a joué un rôle décisif dans le développement de sa poétique et dans sa décision de se distancer du genre de christianisme avec lequel il avait été élevé. Mais il y a plus que cette affinité générale. Étant donné le suffixe «-liba», ce passage de Voltaire pourrait être un cas d'intertextualité, car il semble avoir indirectement contribué à la description du «pays de Molloy » par Beckett dans le roman Molloy et à sa décision d'appeler cette région «Ballyba», la région autour du bourg de «Bally». Dans un autre roman de Beckett, L'Innommable, le texte suggère que Bally est le lieu où la vie a été imposée à l'innommable, «endroit qui, à les en croire, m'aurait infligé le jour64». Dans le manuscrit, cet endroit s'appelle «Baile atha Cliath», nom irlandais de Dublin, suivi de la clause de mise en garde ludique entre parenthèses : «(je ne garantis pas l'orthographe) ${ }^{65}$ ».

En plus de ce lien avec le pays natal de Beckett, la Bible italienne et le lien indirect avec Voltaire ajoutent quelques dimensions intéressantes aux noms de Bally et de Ballyba : ils sont certainement inspirés de Dublin, mais peut-être aussi d'Oolla et d'Ooliba. Dans Ézéchiel 23, on lit que la parole de Dieu s'est adressée au prophète, en parlant de ces deux sœurs qui se prostituent auprès des Égyptiens, des Assyriens et des Chaldéens. Le Dieu d'Ézéchiel n'est pas particulièrement subtil dans son développement de la métaphore. À un moment donné, il insiste pour que son message soit clair en donnant explicitement la clé de la métaphore ou de l'allégorie : «Oolla, c'est Samarie; Ooliba, c'est Jérusalem.» (Ézéchiel 23:4). Ainsi, Oolla représente la capitale d'Israël et Ooliba la capitale de Juda. En hébreu, Oolla signifie «sa propre tente », Ooliba signifie «ma tente est en elle» (car la maison de Dieu se trouve à Jérusalem). Dans le roman Molloy, la nature allégorique de Bally et Ballyba est similaire, bien qu'elle soit tordue. La description de l'énorme libido de Oolla et Ooliba dans le récit d'Ézéchiel est aussi hyperbolique que la description de Bally et Ballyba par le narrateur (Moran), en particulier

62. Voltaire, Euvres complètes, t. XXI, Paris, Garnier, 1879, p. 456.

63. Ibid., p. 457.

64. Samuel Beckett, L'Innommable, Paris, Minuit, 1953, p. 21.

65. Beckett Digital Manuscript Project, BDMP3, MS-HRC-sB-3-10, 5 vo. 
sa description de l'économie fécale de Ballyba, basée sur les «selles de ses citoyens ${ }^{66}$ ». Ballyba est gouverné par quelqu'un qui vit et porte des vêtements blancs comme le pape, et qui s'appelle «Obidil» (anagramme de «libido»).

Cet exemple n'est évidemment qu'un détail, mais il montre que la bibliothèque d'un auteur peut enrichir nos interprétations des œuvres. La recherche génétique a été perçue comme une stratégie de restriction interprétative : par exemple, comme Lernout le suggère, limiter ses interprétations à ce qui peut être falsifié, à ce qui donne à d'autres chercheurs la possibilité de contredire son interprétation (cf. supra); ou tenter de limiter ses interprétations au moyen de ce que Louis Hay a appelé «l'attestation d'une trace scripturaire ${ }^{67}$ ». Mais la recherche exogénétique est plus que ça. En tant que stratégie de lecture, elle ne réduit pas seulement et pas nécessairement l'interprétation. La bibliothèque n'est pas une base de données contenant des faits précis; elle nécessite sa propre stratégie de lecture herméneutique. Dans le cas de l'exemple ci-dessus, les nouvelles informations exogénétiques font de Bally plus qu'une simple référence à Dublin et, par extension, au pays d'origine de Beckett. Ainsi, Bally obtient également une dimension religieuse, et puisque Molloy a été écrit dans la langue de Voltaire peu après que Beckett eut décidé de commencer à écrire systématiquement en français. Nous avons donc trois éléments qui, à leur tour, suggèrent une dimension joycienne intertextuelle sous-jacente, puisque ce sont les trois éléments que Stephen Dedalus essaie de fuir dans A Portrait of the Artist As a Young Man: «You talk to me of nationality, language, religion. I shall try to fly by those nets 68 .»

Cependant, comme Beckett l'a écrit dans la première phrase de son essai sur Joyce, le danger réside dans la netteté des identifications, et Beckett n'est évidemment pas Joyce. Après l'état dactylographié, il a supprimé la description de l'économie fécale de Ballyba, mais non sans laisser une cicatrice textuelle. Lorsque, dans la deuxième partie de Molloy, le narrateur est sur le point de raconter son histoire sur l'économie de Ballyba, il change soudainement d'avis et dit: «Non, je ne vous dirai rien» («No, I'll tell you nothing69»). Cette forme de «reprise» est devenue un trait distinctif de l'écriture de Beckett, qui se caractérise par une intertextualité invisible. Alors que, dans Ézéchiel 23, Dieu insiste pour que son message soit rendu aussi explicite que possible, Beckett a tendance à rendre tout de plus en plus implicite dans son œuvre qui procède de l'érosion.

\section{Stratégie 5 : une approche épigénétique}

Comme indiqué ci-dessus, l'exogenèse peut affecter non seulement l'endogenèse, mais également l'épigenèse. Le dialogue de Beckett avec Paul Verlaine est un bon exemple, à la fois d'une interaction exo/endogénétique et d'une interaction exo/épigénétique. Quelques livres dans la bibliothèque de Beckett contiennent des fragments de papier avec des listes.

66. Samuel Beckett, manuscrit de Molloy, Beckett Digital Manuscript Project, BDMP4, MS-HRC-SB-4-7, p. 66 ro. 67. Louis Hay, «Critiques de la Critique Génétique», Genesis, no 6, 1994, p. 11-23 : «après avoir renoncé à "lire dans les âmes", à revivre l'expérience intérieure de l'écrivain, la génétique a pu se donner une position critique autonome : elle vise les processus d'écriture dans la réalité de leur exécution, dans l'attestation d'une trace scripturaire», en ligne sur persee.fr.

68. James Joyce, A Portrait of the Artist as a Young Man, Londres, Penguin, 1992, p. 220.

69. Beckett Digital Manuscript Project, BDMP4, MS-HRC-SB-17-6, fo 214 ro. 
Par exemple, un «top cinq» de poèmes de Verlaine, écrit sur un bout de papier, est inséré dans son exemplaire non annoté des EEvres poétiques complètes du poète dans l'édition de la Pléiade ${ }^{70}$. Beckett a acquis ce livre assez tard dans sa vie (1968), mais cela n'implique pas qu'il ait lu Verlaine seulement après 1968. Il l'avait sûrement étudié au Trinity College de Dublin (un des livres de Verlaine figurait sur la liste des lectures obligatoires), mais d'une part, en ce temps-là, il n'avait pas encore les moyens d'acheter tous les livres qu'il lisait (il les empruntait à la bibliothèque), et d'autre part, parmi ses livres, plusieurs ont ensuite été vendus ou donnés à des amis. En conséquence, il est difficile de dire à quoi correspond exactement «le top cinq», s'il représente les poèmes préférés de l'auteur en tant que lecteur âgé de soixante ans ou s'il s'agit d'une reconstruction par cet artiste sexagénaire des poèmes qu'il aimait quand il était encore un jeune homme.

Nous savons que, jeune homme, Beckett a mémorisé plusieurs poèmes et connaissait probablement au moins une partie de la poésie de Verlaine par cœur. Au moment où il écrivait le roman Molloy, peu après la Seconde Guerre mondiale, il se souvenait encore de sa lecture de Verlaine. Dans le manuscrit français, quand le personnage éponyme, Molloy, s'éloigne de la voix qu'il entend, la voix en question est clairement celle de Molloy, autrement dit, il s'entend lui-même : «J'écoute et m'entends dicter un monde figé en perte d'équilibre ${ }^{71}$.» Mais Beckett a ensuite biffé ce passage. Cet acte de raturer coïncide avec la fin d'une session d'écriture. Dans les versions suivantes, il devient de moins en moins clair si la voix est vraiment la sienne. Beckett a commencé à réécrire le passage sur une nouvelle page (fo 25 ro), en indiquant la date «7.8.47» au verso (fo 24 vo). La voix devient un murmure, rien qu'un souffle et le manuscrit donne un indice rare sur l'origine de cette allusion :

Et j'écouterais encore < cette chère voix ce cher souffle > lointaine, depuis longtemps tue (on dirait du Verlaine) et que j'entends enfin $>72$.

Le texte fait référence à la première section («Mélancolie») des Poèmes saturniens (1866) de Paul Verlaine, plus précisément au sonnet «Mon rêve familier», qui s'ouvre et se termine par les strophes suivantes :

Je fais souvent ce rêve étrange et pénétrant

D'une femme inconnue, et que j'aime, et qui m'aime

Et qui n'est, chaque fois, ni tout à fait la même

Ni tout à fait une autre, et m'aime et me comprend.

[...]

Son regard est pareil au regard des statues,

Et, pour sa voix, lointaine, et calme, et grave, elle a

L'inflexion des voix chères qui se sont tues ${ }^{73}$.

70. Beckett Digital Library, https://www.beckettarchive.org/library/VER-OEU.html. Paul Verlaine, Euvres poétiques complètes, Paris, Gallimard, 1968; Dirk Van Hulle et Mark Nixon, Samuel Beckett's Library, Cambridge, Cambridge UP, 2013, p. 61.

71. Samuel Beckett, manuscrit de Molloy, Beckett Digital Manuscript Project, BDMP4 MS-HRC-SB-4-6, fo 24 ro. 72. Beckett Digital Manuscript Project, BDMP4 MS-HRC-SB-4-6, 24 vo-25 ro.

73. Beckett Digital Library, https://www.beckettarchive.org/library/VER-OEU.html. Paul Verlaine, Euvres poétiques complètes, op. cit. 
Le manuscrit de Molloy est la seule version dans laquelle Beckett ajoute la parenthèse avec la référence explicite «(on dirait du Verlaine)». Dans les versions suivantes, il atténue la trace exogénétique, de sorte que dans la version publiée et particulièrement dans sa propre traduction anglaise, le poème est à peine reconnaissable en tant que référence intertextuelle :

Et j'écouterais encore ce souffle lointain, depuis longtemps tu et que j'entends enfin

[And if I went on listening to that far whisper, silent long since and which I still hear, I would learn still more, about this $\left.{ }^{74}\right]$.

Mais grâce au manuscrit et à la liste glissée dans l'exemplaire de la Pléiade, nous savons que c'est une allusion au numéro quatre du «top cinq».

Plus tard, en 1977, alors que Beckett travaillait sur Solo (sa traduction française de A Piece of Monologue), il se souvenait encore de Verlaine. Tout en révisant le manuscrit dactylographié de Solo ${ }^{75}$, il se demandait comment traduire le mot «gone» dans «All gone so long 76 ». Le protagoniste regarde un mur sur lequel avaient été accrochées plusieurs images :

Debout donc face au mur. Surface blanche dans l'ombre. Blanche jadis. Grêlée de piqûres d'épingle. Jadis à chaque vide un visage. Là son père. Ce vide grisâtre. Là sa mère. Là tous les deux. Souriants. Jour des noces. Là tous les trois. Cette tache grisâtre. Là tout seul. Lui tout seul. Plus maintenant. Oubliés. En allés 77 .

Dans le texte dactylographié révisé, la traduction originale de «All gone so long» était «Depuis si longtemps ela en allés 78 ». Beckett l'a souligné, a entouré «en allés» et a écrit dans la marge supérieure :

(V.) une âme en allée Vers d'autres cieux

(G.) je me souviens des heures en allées 79 .

La première note marginale fait référence à l'«Art poétique» de Verlaine, dont l'avant-dernière strophe se lit comme suit :

De la musique encore et toujours!

Que ton vers soit la chose envolée

Qu'on sent qui fuit d'une âme en allée

Vers d'autres cieux à d'autres amours ${ }^{80}$.

La deuxième note marginale du manuscrit de Beckett fait référence au livre VIII des Nourritures terrestres d'André Gide :

Je me souviens des heures en allées; pieds nus sur les dalles; j'appuyais mon front au fer mouillé du balcon; sous la lune, l'éclat de ma chair comme un fruit merveilleux à cueillir81.

74. Samuel Beckett, Molloy, Paris, Olympia Press, 1955, p. 53.

75. Id., tapuscrit de Solo, MS UoR 2604, fo 03 ro.

76. Id., tapuscrit de A Piece of Monologue, MS UoR 2070, fo $02 \mathrm{r}^{\circ}$.

77. Id., «Solo», dans Catastrophe et autres dramaticules, Paris, Minuit, 1982, p. 12.

78. Id., tapuscrit de Solo, MS UoR 2604, fo 03ro.

79. Ibid.

80. Paul Verlaine, «Art poétique», Jadis et Naguère, Paris, Léon Vanier, 1884, p. 24.

81. André Gide, Les Nourritures terrestres, Paris, Mercure de France, 1897, p. 199. 
Tout en révisant sa traduction du simple mot «gone», Beckett a donc consulté deux sources littéraires de son immense entrepôt mental de citations littéraires. Dans le manuscrit précédent, il avait déjà choisi «en allés », ce qui suggère que les deux citations ne servaient pas tant de support lexical pour la traduction, mais qu'elles témoignent plutôt de la tentative de l'auteur de placer sa traduction dans une relation intertextuelle avec la tradition littéraire de la langue cible. En ce sens, il témoigne également de la «condition intertextuelle»des œuvres de Beckett.

Comme le montrent les deux exemples relatifs à Verlaine, Beckett ne fait pas de distinction claire entre la genèse pré-éditoriale et la genèse post-éditoriale en ce qui concerne l'utilisation de matériaux exogénétiques. L'exogenèse ne se limite pas à la genèse avant le moment du «bon à tirer» et n'interagit pas uniquement avec l'endogenèse. Comme il n'y a pas de frontière précise entre l'exogenèse et l'endogenèse (car dès qu'un auteur écrit une note de lecture, elle fait déjà potentiellement partie de l'endogenèse) ${ }^{82}$, cette dialectique se situe aussi entre exo- et épigenèse. Une dynamique triangulaire semble donc appropriée pour modéliser l'interaction entre exo-, endo et épigénèse, en montrant comment, à tout moment du processus d'écriture, l'exogenèse peut agir non seulement sur l'endogenèse, mais également sur l'épigénèse.

\section{Conclusion : intertextualité invisible comme dialectique négative}

Outre le «code linguistique» et le «code bibliographique» de la «condition textuelle» telle que définie par McGann83, la condition intertextuelle implique, comme indiqué plus haut, un «code génétique». L'analyse de ce code peut être très utile dans des cas d'intertextualité invisible - ou plutôt inouïe, comme dans le cas de la voix lointaine de Verlaine. Raymonde Debray Genette a proposé de définir ce code en distinguant l' «endogenèse » de l' «exogenèse ». Mais tandis que l'endogenèse correspond à l'«intérieur» du processus d'écriture, l'exogenèse n'est qu'un seul aspect de l'«extérieur», dont l'épigenèse représente un autre aspect. L'exogenèse est constituée de sources externes qui existent indépendamment de l'œuvre que l'auteur écrit; dans de nombreux cas, elles existent déjà avant même que l'auteur ait écrit le premier mot. L'épigenèse interagit également avec le monde «externe», mais la différence est que l'œuvre de l'auteur fait déjà partie de ce monde externe. La critique génétique a toujours reconnu que la frontière entre l'endogenèse et l'exogenèse était floue; il est impossible de déterminer exactement où l'une se termine et où l'autre commence (cf. supra). De même, on peut se demander s'il existe une frontière claire entre exogenèse et épigenèse. La relation entre le texte et l'intertexte est une co-dépendance continue, une «dialectique négative» à travers le moment du «bon à tirer».

Dans ce contexte, ma suggestion serait d'interpréter l'intertextualité invisible dans le sens que Theodor Adorno a donné à la «dialectique négative», et que Paul Eggert a appliqué à «l'œuvre» dans le contexte des études textuelles. Étant donné que les lecteurs doivent constamment donner vie à un document contenant le texte d'une œuvre littéraire, Eggert voit l'œuvre en termes de dialectique négative entre le support matériel (la dimension

82. Raymonde Debray-Genette, «Génétique et poétique : esquisse de méthode », Littérature, no 28, 1977, p. 19-39; Pierre-Marc de Biasi, «What Is a Literary Draft? Toward a Functional Typology of Genetic Documentation », Yale French Studies, no 89, 1996, p. 26-58.

83. McGann, op. cit., p. 52. 
documentaire) et l'expérience significative (la dimension textuelle) ${ }^{84}$. La dialectique qui constitue cette dynamique fondamentale est «négative» parce qu'il ne peut y avoir de fin définitive. Au lieu de l'espoir d'une transcendance positive ou Aufhebung, le seul espoir qu'offre la «dialectique négative» d'Adorno est qu' «elle ne se repose pas en elle-même» : «Il appartient à la détermination d'une dialectique négative de ne pas se reposer en elle-même, comme si elle était totale. C'est là sa figure d'espérance 85 .» De même, l'intertextualité invisible ne se repose pas en elle-même. Il faut des éditions génétiques et des lecteurs informés des manuscrits pour lui donner vie, et nous avons dès lors besoin d'un ensemble de stratégies de lecture, au développement desquelles cette contribution a tenté de donner une impulsion.

84. Paul Eggert, Securing the Past: Conservation in Art, Architecture and Literature, Cambridge, Cambridge University Press, 2009, p. 236-237.

85. Theodor W. Adorno, Negative Dialectics, trans. E.B. Ashton, Londres, Routledge \& Kegan Paul, 1973, p. 406 
DiRK VAN HULLE est professeur de bibliographie et d'histoire du livre à l'Université d'Oxford et directeur du Centre pour la génétique des manuscrits (Centre for Manuscript Genetics, Université d'Anvers). Il a publié Textual Awareness, Modern Manuscripts, Samuel Beckett's Library (avec Mark Nixon), The New Cambridge Companion to Samuel Beckett, James Joyce's Work in Progress, sept volumes de la série «Making of» (Bloomsbury) et plusieurs éditions génétiques au sein du Beckett Digital Manuscript Project, y compris la Beckett Digital Library.

dirk.vanhulle@uantwerpen.be

\section{Stratégies de lecture exogénétiques : approches de l'intertextualité invisible}

Cet article tente d'évaluer les tensions et les correspondances entre exogenèse et intertextualité, en abordant notamment l'érosion intertextuelle et le phénomène selon lequel un intertexte agit sur la genèse d'un texte, mais reste invisible dans sa version publiée - ce qu'on pourrait appeler l'intertextualité invisible. J'examine dans quelle mesure l'exogenèse diffère de la «critique des sources» (que Julia Kristeva rejetait comme un sens banal et mal compris d'intertextualité) et propose cinq stratégies de lecture exogénétiques pour traiter de l'intertextualité invisible : une approche exogénétique directe, une approche endogénétique, une approche intratextuelle, une approche indirecte via les marginalia et une approche épigénétique.

This essay examines the tensions and correspondences between exogenesis and intertextuality, principally by focusing on intertextual erosion and on the phenomenon of how an intertext impacts the genesis of a text but remains out of sight in the published version - a phenomenon that could be called "invisible intertextuality". The essay investigates the extent to which exogenesis differs from "source criticism" (which Julia Kristeva rejected as a banalization and misinterpretation of intertextuality), and it suggests five exogenetic reading strategies to deal with invisible intertextuality: a direct exogenetic approach, an endogenetic approach, an intratextual approach, an indirect approach via marginalia, and an epigenetic approach.

In diesem Artikel wird versucht, die Spannungen und Korrespondenzen zwischen Exogenese und Intertextualität zu bewerten, insbesondere durch die Behandlung der intertextuellen Schwächung, womit ein Intertext auf die Entstehung eines Textes einwirkt, aber in seiner veröffentlichten Fassung unsichtbar bleibt - was man als unsichtbare Intertextualität bezeichnen könnte. Ich untersuche, inwiefern sich die Exogenese von der „Quellenkritik“ (die Julia Kristeva als banale und missverstandene Intertextualität ablehnte) unterscheidet, und schlage fünf exogenetische Lesestrategien für den Umgang mit unsichtbarer Intertextualität vor : einen direkten exogenetischen Ansatz, einen endogenetischen Ansatz, einen intratextuellen Ansatz, einen indirekten Ansatz über die Marginalien und einen epigenetischen Ansatz.
Este artículo intenta evaluar las tensiones y las correspondencias entre exogénesis e intertextualidad, considerando en particular la erosión intertextual y el fenómeno según el cual un intertexto afecta la génesis de un texto, pero permanece invisible en su versión publicada - es lo que se podría denominar la "intertextualidad invisible". Analizo en que medida la exogénesis difiere de la "crítica de las fuentes" (que Julia Kristeva rechazaba en tanto que sentido trivial y mal entendido de la intertextualidad) y propongo cinco estrategias de lectura exogenética para abordar la intertextualidad invisible : un enfoque exogenético directo, un enfoque endogenético, un enfoque intratextual, un enfoque indirecto a través de las marginalia y un enfoque epigenético.

Este artigo procura avaliar as tensões e correspondências entre exogénese e intertextualidade, abordando designadamente a erosão intertextual e o fenómeno através do qual um intertexto age sobre a génese de um texto, mas permanece invisível na versão publicada - o que se poderia chamar intertextualidade invisível. $\mathrm{O}$ artigo investiga em que medida exogénese difere de "crítica das fontes" (que Julia Kristeva rejeitava como sentido banal e má interpretação de intertextualidade) e propõe cinco estratégias de leitura exogenética para lidar com a intertextualidade invisível : uma abordagem exogenética direta, uma abordagem endogenética, uma abordagem intratextual, uma abordagem indireta via marginalia e uma abordagem epigenética.

L'articolo tenta di valutare le tensioni e le corrispondenze tra esogenesi e intertestualità, affrontando soprattutto l'erosione intertestuale e il fenomeno secondo il quale un intertesto agisce sulla genesi di un testo ma resta invisibile nella sua versione pubblicata - cosa che si potrebbe chiamare l'intertestualità invisibile. Il saggio analizza in che misura l'esogenesi differisce della "critica delle fonti" (che Julia Kristeva rigettava in quanto senso banale e frainteso d'intertestualità) e propone cinque strategie di lettura esogenetica per trattare dell'intertestualità invisibile : un approccio esogenetico diretto, un approccio endogenetico, un approccio intratestuale, un approccio indiretto attraverso i marginalia e un approccio epigenetico. 